\title{
The difference of a smart diaper for special needs adults
}

\begin{abstract}
There are many adult diaper users who are unable to recognize when they have soiled themselves. Some diapers approach the problem by integrating smart technology into the textile to assist users. Five smart diapers were selected to be evaluated and compared their capacity to service the needs of consumers. All smart diapers were intended to be not only help serve special needs patients, but also help the caregivers to provide a quicker and more tuned in service. This project is an attempt to compare and contrast these five smart diapers and give caregivers the ability to make informed purchases relative to the special needs of their clients.
\end{abstract}

Keywords: smart diaper, consumers, adult diaper, textile industry, wetness perception
Volume 3 Issue 4 - 2017

\author{
Shu Hwa Lin,' Kaimi Kajiyama,' Tien Hsiang \\ $\mathrm{Wu}^{2}$ \\ 'University of Hawaii at Manoa, USA \\ ${ }^{2}$ Sinopulsar Technology Inc, Hsinchu City, Taiwan
}

Correspondence: Shu Hwa Lin, Associate Professor,

Department of Family \& Consumer Sciences, University of Hawaii at Manoa, Honolulu, HI 96822, USA, Tel 808956 2245, Email shulin@hawaii.edu

Received: November 27, 2017 | Published: December II, 2017

\section{Introduction}

There are a variety of consumers who benefit from adult diapers. Adult diapers help serve individuals who face daily problems, whether it is those with dementia, urinary/fecal incontinence, diarrhea or experience mobility impairment. Being unable to have complete autonomy over your body's actions can be a humiliating and embarrassing experience. Not only that, but should a person's diaper go unattended, it can lead to even greater health concerns. Market research suggests adult diaper sales have exceeded 1.5 billion annually in the health hygiene market. ${ }^{1}$ A contributing factor to this is the way care facilities approach the issue. They opt for inexpensive, less absorbent diapers and instead change at a higher frequency.

The smart diaper is not only for convenience, but to offer assistance to patients who are unable to have complete control over their bodily functions. Many people face the challenges of incontinence, and the smart diaper helps to better serve their physiological needs. With over 13 million Americans alone suffering from urinary incontinence, this diaper offers a more sustainable alternative. ${ }^{2,3}$ This diaper helps to give greater accuracy to the level of moisture in the diaper, thus reducing the level of waste. It does not pose health complications to patients who aren't carefully monitored nor have a high frequency of changing such as diaper rash. While there are alternatives, such as catheters, many people who suffer from incontinence cite that they prefer less invasive means. ${ }^{3,4}$ In addition, unlike other diapers with smart technology, this diaper can alert the care taker about fecal excrement.

Through integrated technology, the smart diaper is able to sense and detect the moisture level of the diaper. Taking it even further, with the use of smart phone technology, an app can easily communicate to the caregiver about the status of the diaper and allow for closer monitoring. Also, the data collected from the app can give greater understanding into the problem, as well as how new solutions can help better alleviate the issue. The clothing and textile industry are greatly interested in the study of skin wetness perception (WP) because it significantly affects the consumers comfort level. ${ }^{4-6}$ The knowledge that can be gained from the smart phone app can help the industry understand the needs of the market, as well as better statistics and demographics on the adult diaper target consumer.

Care givers or diaper users desire special functions that can alleviate tasks related to monitoring the patient, such as: accuracy measure position, urinary sense, faces sense, alert, real-time observation, record excrete pt., anti-lost, predict excrete, and predict health. A huge component to the disconnect between patient and caretaker is that the caretaker isn't able to actively recognize when their patients need service. The smart diaper features an app that monitors their patient's status in real time, bridging the divide between caretaker and patient significantly.

When weighing the functions and capabilities the smart diaper offers compared to other e-diapers, the smart diaper can offer a greater range of services, and therefore, be more marketable to a greater audience. The broad range of applications of the smart diaper is effective for a community that can have a wide range of intersecting disabilities, whether it is immobility, dementia, etc. The target demographic of people who are in need of this service would be more inclined to have a product that offers a variety of solutions rather than one that limits their extent of care.

\section{Smart diaper: target consumers for special needs}

All adult diapers in the market are served as protection. This project is an attempt to evaluate five smart diapers on the following qualities:

a. Dementia Patients

Easy to manage at the right time:

i. Disabled people who can't move lower torso

ii. Immobile people who can't go to bathroom by themselves

\section{Smart diaper comparison}

A total of five diapers were selected; including three smart or E-diapers, one color diaper and convention diapers; and evaluated 10 functions listed on Table 1. A smart diaper should have special 
characteristics for modern life, such as: accuracy measure position, urinary sense, faces sense, alert, real-time observation, record excretes pt., anti-lost, predict excrete, and predict health. Conventional diapers only provide the most basic capabilities, only acting as a temporary solution to a person's bodily excretions. They don't have any sensing or IoT capabilities that many of the smart textile diapers offer to relieve caregivers of tasks.

Some diapers offer the ability to sense moisture through chemical change in color after soil. Rather than an indication through change in color on the diaper, the smart diaper can actively notify you of changes. The color-changing diaper: The function of the colorchanging diaper is to have a chemical agent on the inside of the batch. When the humidity detected in high inside of the diaper, the diaper will change color. While the color-changing diaper changes color to indicate soil, it doesn't indicate when soil occurred. It offers a passive alert to caretakers rather than an active one that alerts them to concerns.

On the market are diapers with Bluetooth capabilities. Some are built-in, whereas others allow for a Bluetooth accessory to be added in conjunction. Where it succeeds the color-changing diaper in actively alerting caretakers in real time, it falls short in its ability to recognize

Table I Comparison of capacities of smart diaper products excrement. It also doesn't offer the broader capabilities the smart diaper offers, limiting its range of consumers it can offer services to.

The smart diaper, in comparison to its competitors, not only expands on the functional aspects few other diapers offer, but also integrates different software and capabilities as a mode of communication between the caregiver and patient. In particular, the mobile app integrates technology that not only can display the status of moisture in the diaper, but also account for the difference between soil and excrement, alert caregivers and be optimized at custom settings for a finer tuned experience. The smart diaper has a built in GPS tracking function, a service beneficial to dementia patients. The RF signal of the sensing module offers anti-loss capabilities for the care receiver, in the event that the patient could get lost.

The smart diaper addresses the issue of patients going unnoticed by essentially connecting the patient to the caregiver through a mobile app. Rather than facing the possibility of diaper rash, complaints from patients who are aware and the consequences of patients who would be unaware, the smart diaper allows for caretakers to be more informed about the status of their patients. Through the use of both sensors and the mobile app, the connection between patient and caretaker can become so much more integrated (Table 1).

\begin{tabular}{|c|c|c|c|c|c|}
\hline Tested diaper & Smart diaper & E-diaper I & E-diaper 2 & Color diaper & $\begin{array}{l}\text { Convention } \\
\text { diaper }\end{array}$ \\
\hline Photo & $=\times 1=$ & & & & \\
\hline Diaper type & Diaper +Blue tooth & Blue tooth & Diaper +Blue tooth & Diaper & Diaper \\
\hline \multirow{2}{*}{ Accuracy measure position } & High & Low PE/Outside & & Low PE/Inside & None \\
\hline & Skin surface & & PE/Inside & & \\
\hline Urinary sense & Yes & Yes & Yes & Yes & No \\
\hline Feces sense & Yes & No & No & No & No \\
\hline Type of alert & Active & Active & Active & Color/Passive & No \\
\hline Real-time & Yes & Yes & Yes & No & No \\
\hline Record excrete pt. & Yes & No & No & No & No \\
\hline Anti-lost & Yes & Yes & Yes & No & No \\
\hline Predict excrete & Yes & No & No & No & No \\
\hline Predict health & Yes & No & No & No & No \\
\hline
\end{tabular}

The use of mobile health apps are on the rise in demand, and expanding annually. Not only can it makes the healthcare experience more involved and interactive, but also allows both doctors and patients to keep track of their health as well as their health history. It also can help the healthcare industry through the data it collects on effectiveness in care and help better assess the consumer. Integrated technology is playing a bigger role in so many industries, and its involvement is only going to be more deeply intertwined. By adopting the smart diaper, caregivers could be reducing the amount of checking and trying and cut down on waste from dispensable conventional diapers. The illustration in Figure 1 demonstrates the process of the smart diaper in the nursing cycle of diaper changing. Caregivers can observe the moisture in diaper by using mobile phone apps (Figure 2). 


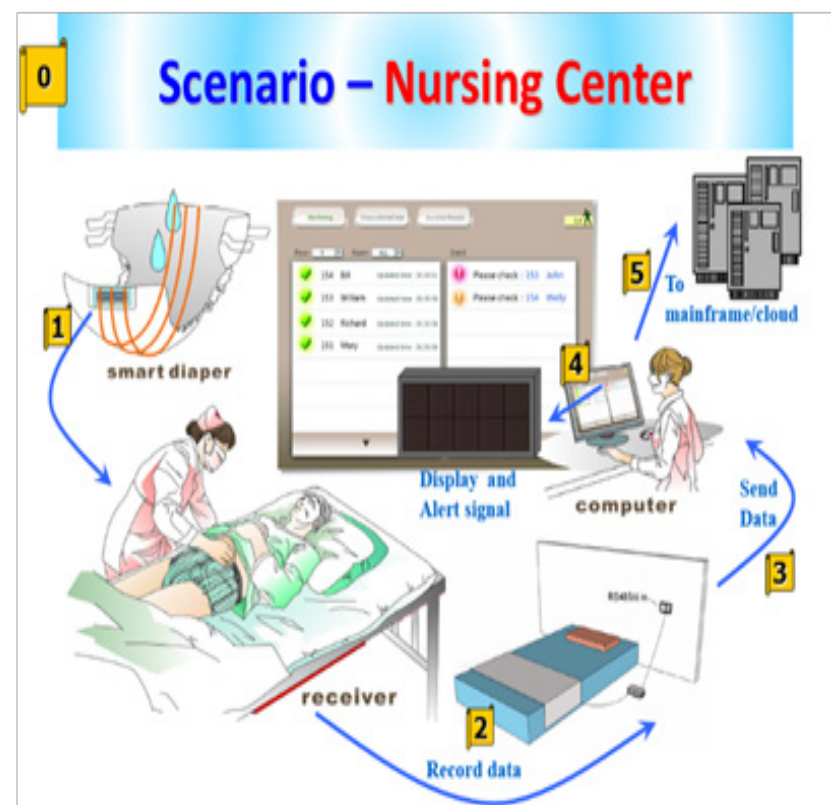

Figure I Smart Things - Assistance for Ambient Assisted Living. This illustration demonstrates the process of the smart diaper.

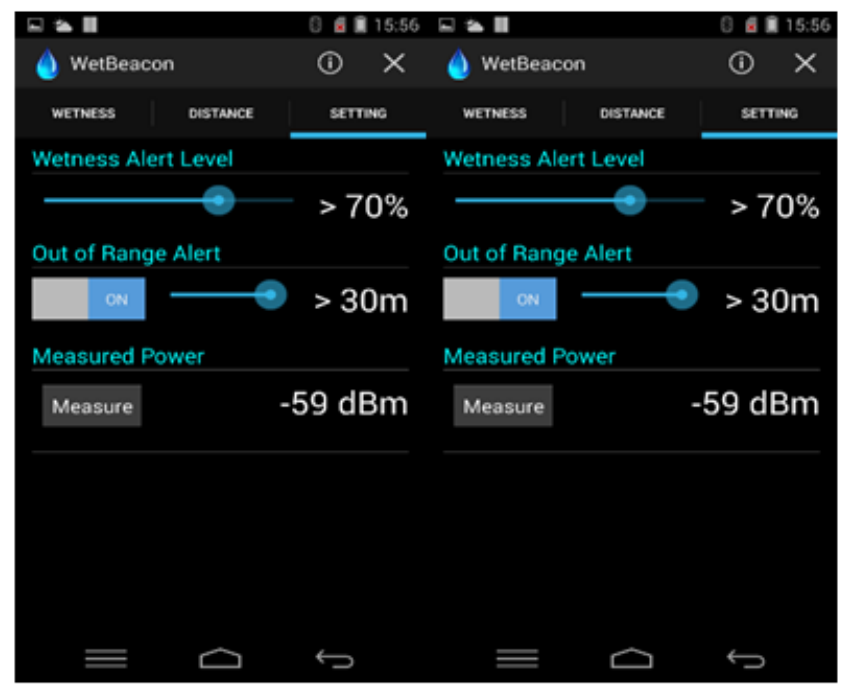

Figure 2 Two screen shoots: the smart phone APP can be configured to alert you when there is a significant change in moisture.

\section{Materials/equipment}

Like the conventional diaper, the smart diaper consists of 3 basic layers, an outer shell of breathable polyethylene film or a nonwoven and film composite which prevents wetness and soil transfer, an inner absorbent layer of a mixture of air-laid- paper and superabsorbent polymers for wetness, and a layer nearest to the skin, made of nonwoven materials (where smart diaper adds wired-sensors) with a distribution layer directly beneath which transfers wetness to the absorbent layer (Figure 3). Expanding on the conventional diaper, the other diapers feature a color changing chemical agent or bluetooth devices, either built in or separately, connected through wired sensors. In addition to these features, the smart diaper also can connect to the smartphone via a mobile app.
Three wired-sensors are installed to cover all possible areas to detect moisture. Smart Diaper includes:

a. Wet Beacon Module (Figure 4),

b. App for Smart Phone,

c. Wet Observer (optional)

d. Sensing Diaper (Figure 3).

The item of 1 3 are reusable, and item 4 sensing diaper is not a reusable item. The sensors are able to detect moisture by a chemical reaction between the aluminum sensor and the soil. This triggers a signal to the Bluetooth device.

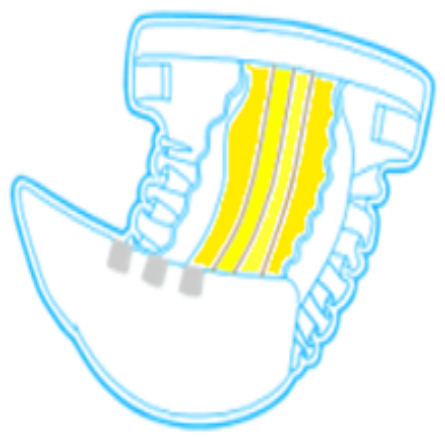

Figure 3 Three wired-sensors are installed to cover all possible area to detect moisture.

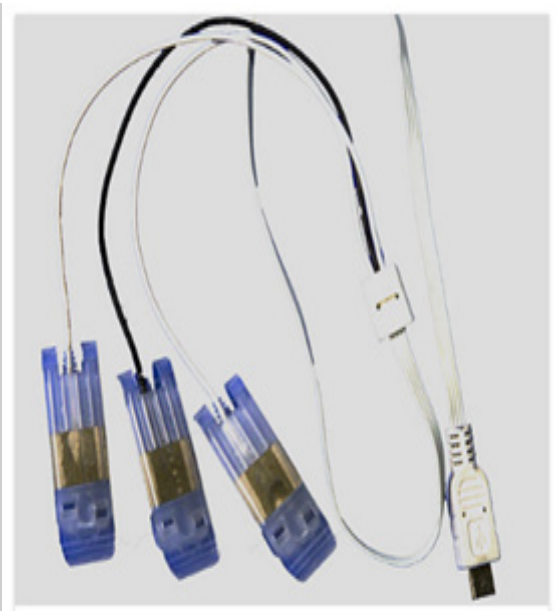

Figure 4 These sensor clips are attached to the aluminum sensors and can be reused for multiple uses.

\section{Discussion and conclusion}

The smart diaper system can help get information about diaper usage; its future data collected is quite important for caregivers to handle changing of the diaper. Understanding the market allows for both smarter investments as well as a more precise service offered. Caregivers may be able to predict or to determine whether diapers are dirty and when to change dirty diapers, as well as to predict when the care receivers might urinate or defecate. This is something not readily offered on the market. The smart diaper system detects moisture in the diaper and is intuitive to the physiological signals of the unconscious actions of the user's body, via the collection of excretions (such as, urine, feces, etc.) from the care receiver. ${ }^{7,8}$ Through the waveform- 
like impedance change, the time and duration of each urination are recorded and analyzed; and consequently, the number of times and frequency of urination, as well as the amount of urine, etc. are obtained. As a result, detection, determination and prediction of the health condition of the care receiver, such as urination frequency or urinary tract infections, are facilitated. ${ }^{9-11}$

The smart diaper is an advanced consumable diaper, creating better communication between the caregiver and care receiver by ICT and textile technology via a skin emulation structure. Assessing the right time to change the dirty diaper will reduce burden for caregiver and reduce the misery of the care receiver too; When the patient has diaper rash (when skin is infected), the patient will experience pain and discomfort, it will affect their temper, and can persist to around 2 weeks, which is a big burden for both patient and caregiver; The smart diaper can help in preventing the problem from the start, eliminating the consequences that can occur later. The smart diaper offers a solution to some of the problems experienced in home-care and nursing care. All of the data history on the app can be collected from the smart phone in order to provide beneficial care in the future.

It will be an important consumable tool for the healthcare and medical industries, as well as the next generation of smart diaper consumers. It will be the best home care device in the smart city, linked with IoT+system for the future medical care. The use of mobile app technology integrated in the design not only improves upon the functionality of the design, but appeals to the shifting population of the digital age. Baby boomers are at the age where healthcare becomes one of the greatest things their income is spent on, and the upcoming generations will be accustomed to the daily integration of technology in their lives. The greater synchronization between the smart diapers will ultimately offer greater demographic and quantitative data to healthcare industries, greater service offered from caretakers and happier patients who will face less adversity in a problem many face, but often isn't discussed.

\section{Acknowledgements}

We thank all the volunteers, publications support, staff, and authors who wrote and provided helpful comments on previous versions of this document. The authors are grateful for the research financial partially support from the research support administered by the College of Tropical Agriculture and Human Resources, University of Hawaii at Manoa.

\section{Conflict of interest}

Author declares there is no conflict of interest in publishing the article.

\section{References}

1. Ward DB, Fleischer AB, Feldman SR, et al. Characterization of diaper dermatitis in the United States. Arch Pediatr Adolesc Med. 2000;154(9):943-946.

2. Tarbox RS, Williams WL, Friman PC. Extended diaper wearing: Effects on continence in and out of the diaper. J Appl Behav Anal. 2004;37(1):97-100.

3. Wai AAP, Foo SF, Jayachandran M, et al. Towards developing effective Continence Management through wetness alert diaper: Experiences, lessons learned, challenges and future directions. Pervasive Computing Technologies for Healthcare (Pervasive Health), 2010 4th International Conference on-NO Permissions. 2010. p. 1-8.

4. Pfisterer MHD, Johnson TM, Jenetzky E, et al. Geriatric patients' preferences for treatment of urinary incontinence: a study of hospitalized, cognitively competent adults aged 80 and older. $J$ Am Geriatr Soc. 2007;55(12):2016-2022

5. Zisberg A, Gary S, Gur-Yaish N, et al. In-Hospital Use of Continence Aids and New-Onset Urinary Incontinence in Adults Aged 70 and Older. J Am Geriatr Soc. 2011;59(6):1099-1104.

6. Goldberg JM, Buchler DA, Dibbell DG. Advanced hidradenitis suppurativa presenting with bilateral vulvar masses. Gynecol oncol. 1996;60(3):494-497.

7. Shvartzman P, Nasri Y. Urine culture collected from gel-based diapers: developing a novel experimental laboratory method. J Am Board Fam Pract. 2004;17(2):91-95.

8. Lyder CH. Perineal dermatitis in the elderly: a critical review of the literature. J Gerontol Nurs. 1997;23(12):5-10.

9. Raccuglia M, Hodder S, Havenith G. Human wetness perception in relation to textile water absorption parameters under static skin contact. Textile Research J. 2016;87(20):2449-2463.

10. Raccuglia M, Hodder S, Havenith G. Fabric water absorption \& wetness perception. In 7th European Conference on Protective Clothing. 2016. p. 143-144.

11. Boulos MNK, Wheeler S, Tavares C, et al. How smartphones are changing the face of mobile and participatory healthcare: an overview, with example from eCAALYX. Biomed Eng Online. 2011;10:24. 